Case Report

\title{
Early-Stage Goniodysgenetic Glaucoma in a Young Beagle Dog
}

\author{
Osamu Sawamoto ${ }^{1}$, and Ken-ichi Umeoka ${ }^{1}$ \\ ${ }^{1}$ Division of Pharmacology, Drug Safety and Metabolism, Otsuka Pharmaceutical Factory, Inc., 115 Tateiwa, Muya-cho, \\ Naruto, Tokushima 772-8601, Japan
}

\begin{abstract}
Signs of glaucoma such as greenish eyes were observed clinically in a 9-month-old male beagle dog. Histopathologically, the glaucomatous eye was characterized by goniodysgenesis (the absence of a trabecular meshwork) in the filtration angle. Spheroid bodies suggestive of axonal degeneration were observed in the axons posterior to the lamina cribrosa, although cupping of the optic nerve head was not seen. The diagnosis of early-stage glaucoma due to goniodysgenesis was made based on the animal's clinical course and histopathological findings. In addition, narrowing of the episcleral veins, fluid retention in the ciliary processes, and retinal edema were noted. The above findings support the concept that glaucomatous optic neuropathy may be caused by a reduction in blood flow rather than an increase in intraocular pressure. (J Toxicol Pathol 2004; 17: 275-278)
\end{abstract}

Key words: glaucoma, goniodysgenesis, dog

Glaucoma is an ocular disorder that encompasses a group of conditions that have in common optic nerve damage, predominantly as the result of an increase in intraocular pressure (IOP). Glaucoma is classified into primary, secondary, and congenital types in a number of animal species including the $\operatorname{dog}^{1,2}$. Primary glaucoma is a genetic, bilateral condition that occurs without antecedent or concurrent ocular or systemic disease associated with an elevated IOP. In contrast, secondary glaucoma results directly from antecedent or concurrent ocular or systemic disease that results in impaired aqueous humor circulation, outflow, or both ${ }^{3}$. An increase in IOP is considered to result from diabetes, miosis, the characteristics of the aqueous humor, adrenocortical hormone, and nervous control. Furthermore, drug-induced glaucoma, which is a form of secondary glaucoma, may result from the use of specific systemic or topical medications. For example, glucocorticoids frequently induce open-angle glaucoma. It has been reported that acute angle-closure glaucoma is induced by several classes of drugs, including adrenergic agonists, cholinergics, anticholinergics, sulfa drugs, selective serotonin reuptake inhibitors, tricyclic and tetracyclic antidepressants, anticoagulants, and histamine $\mathrm{H}(1)$ and $\mathrm{H}(2)$ receptor antagonists ${ }^{4}$. An increase in IOP

Received: 26 May 2004, Accepted: 17 August 2004

Mailing address: Osamu Sawamoto, Division of Pharmacology, Drug

Safety and Metabolism, Otsuka Pharmaceutical Factory, Inc., 115

Tateiwa, Muya-cho, Naruto, Tokushima 772-8601, Japan

TEL: 81-88-685-1151 FAX: 81-88-684-0553

E-mail: sawamoos@otsukakj.co.jp may also be induced by chemicals with an unclear pharmacologic profile. Therefore, spontaneous glaucoma must be distinguished from glaucoma caused by chemicalrelated toxic effects, and a clear understanding of the histopathologic characteristics of each type of glaucoma is important for differential diagnosis in toxicity studies. In this case report, we describe the histological and immunohistochemical features of early-stage spontaneous glaucoma with goniodysgenesis in a young beagle dog.

Signs of glaucoma were observed clinically in a 9month-old male beagle dog (Covance Research Products, Inc., Cumberland, VA, USA). The animal subsequently received a total parenteral nutrition (TPN) solution intravenously for 4 weeks in a nutritional study. The animal was housed individually in a stainless-steel cage $(700 \times 700$ $\times 700 \mathrm{~mm}$ ). The room was maintained at $23^{\circ} \mathrm{C} \pm 3^{\circ} \mathrm{C}$ and $55 \% \pm 15 \%$ relative humidity, with a 12 -hour light-dark cycle (lights on at 7:00) and an air replacement rate of 13-16 times per hour.

Clinically, at the age of 9 months, both eyes appeared greenish and showed increased ocular tension on palpation, although a buphthalmic globe was not observed, and an increase in IOP was suspected. In addition, a shallow anterior chamber with slight conical protrusion of the iris was observed by ophthalmic examination using a slit-lamp biomicroscope (SL-14, Kowa Company, Ltd., Japan). However, no abnormalities such as cupping of the optic disc were observed by funduscopic examination using a binocular indirect ophthalmoscope (Omega 180, Heine Optotechnik, Germany). Two normal dogs that were used in the same nutritional study served as comparative control 

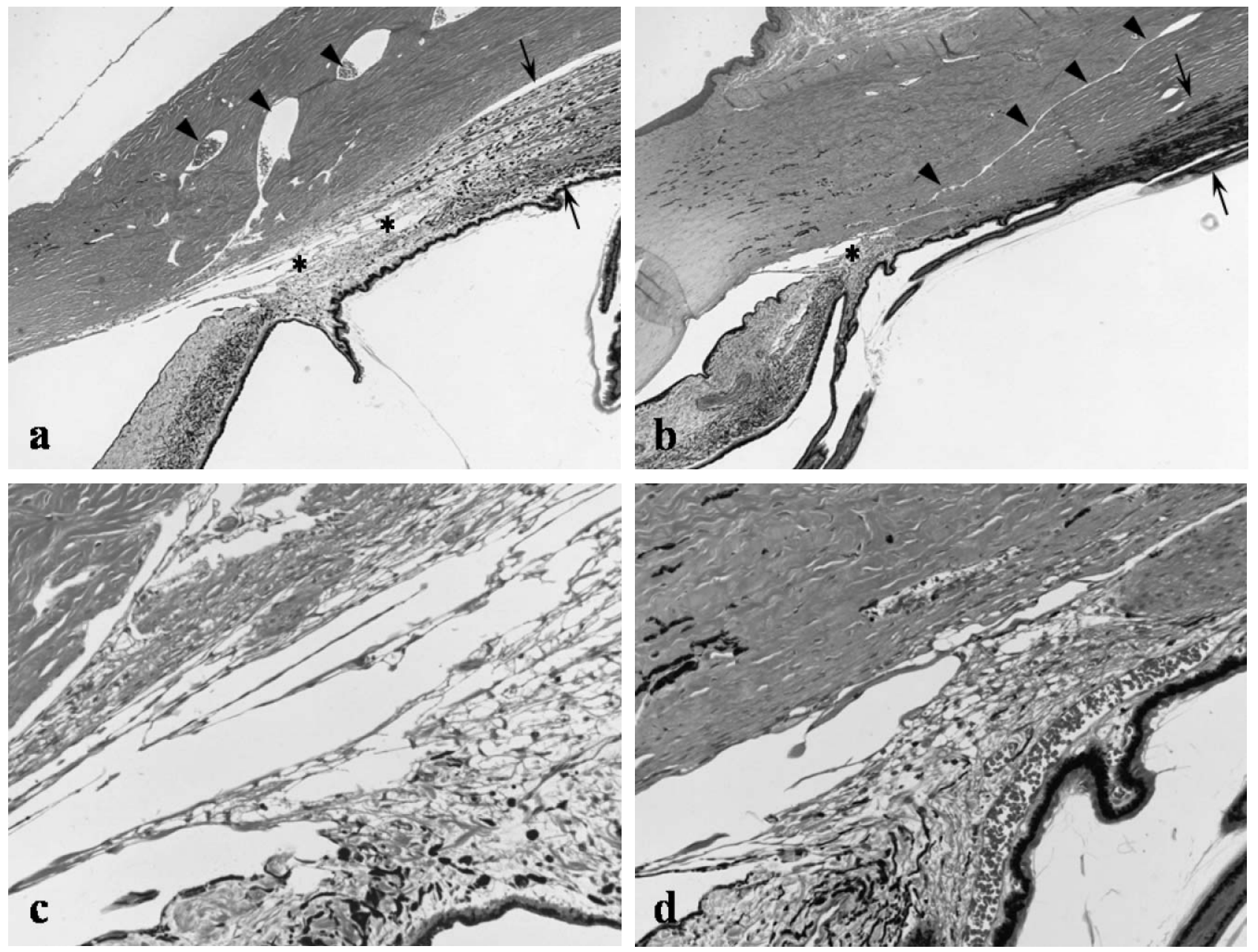

Fig. 1. Filtration angles in a normal $\operatorname{dog}(a, c)$ and a glaucomatous $\operatorname{dog}(b, d)$. (a) The normal filtration angle includes a trabecular meshwork (asterisks), Schlemm's canal, episcleral veins (arrowheads), and the ciliary body (arrows). (b) A scant trabecular meshwork (asterisk), narrowing of Schlemm's canal and the episcleral veins (arrowheads), and a compact ciliary body (arrows) in the glaucomatous dog. (c) Detail of (a): a normal trabecular meshwork. (d) Detail of (b): the scant trabecular meshwork in the glaucomatous dog. H\&E stain, a and $b: \times 30, c$ and $d: \times 150$.

animals. The animals (including the controls) were sacrificed at 10 months of age by exsanguination under pentobarbital sodium anesthesia, and the eyes were removed. These studies were approved by our Institutional Committee on the Guide for the Care and Use of Laboratory Animals, and all procedures were performed in accordance with our institutional guidelines.

The removed eyes were fixed in 3\% glutaraldehyde and $2.5 \%$ formalin, the tissues were embedded in paraffin, and $4-$ $\mu \mathrm{m}$-thick sections were stained with hematoxylin and eosin (H\&E) for microscopic examination. Luxol fast blue (LFB) staining was also performed. In addition, deparaffinized tissue sections were stained immunohistochemically by the labeled streptavidin-biotin (LSAB) method using an LSAB2 kit (DAKO, Carpinteria, CA, USA). The primary antibodies used were rabbit polyclonal antibodies for myelin basic protein (MBP, DAKO) and for glial fibrillary acidic protein (GFAP, DAKO) and mouse monoclonal antibody for neurofilament protein (NF, DAKO).
Histopathologically, goniodysgenesis characterized by a scanty trabecular meshwork in the filtration angle was observed in both eyes of the glaucomatous dog (Figs. $1 \mathrm{~b}$ and $1 \mathrm{~d})$. The root of the iris was located at a more anterior position than in the normal dogs. A narrowed Schlemm's canal was located at a relatively posterior position, and the episcleral veins continuing from Schlemm's canal were also narrowed (Fig. 1b). The ciliary body in this dog was composed mainly of muscle fibers and pigment cells, and loose connective tissue was rarely seen (Fig. 1b). The cystic retention of acidophilic fluid was observed in the ciliary processes. Slight edematous thickening of the ganglion cell layer and nerve fiber layer in the retina was also noted. In the optic nerve, spheroid bodies suggestive of axonal degeneration were seen in the axons posterior to the lamina cribrosa (Fig. 2b). The spheroid bodies in the optic nerve of this dog showed a positive reaction to NF (Fig. 2b, inset), and the optic nerve head was poorly stained by LFB (Fig. $2 \mathrm{c}$ ), whereas the normal optic nerve was stained blue (Fig. 

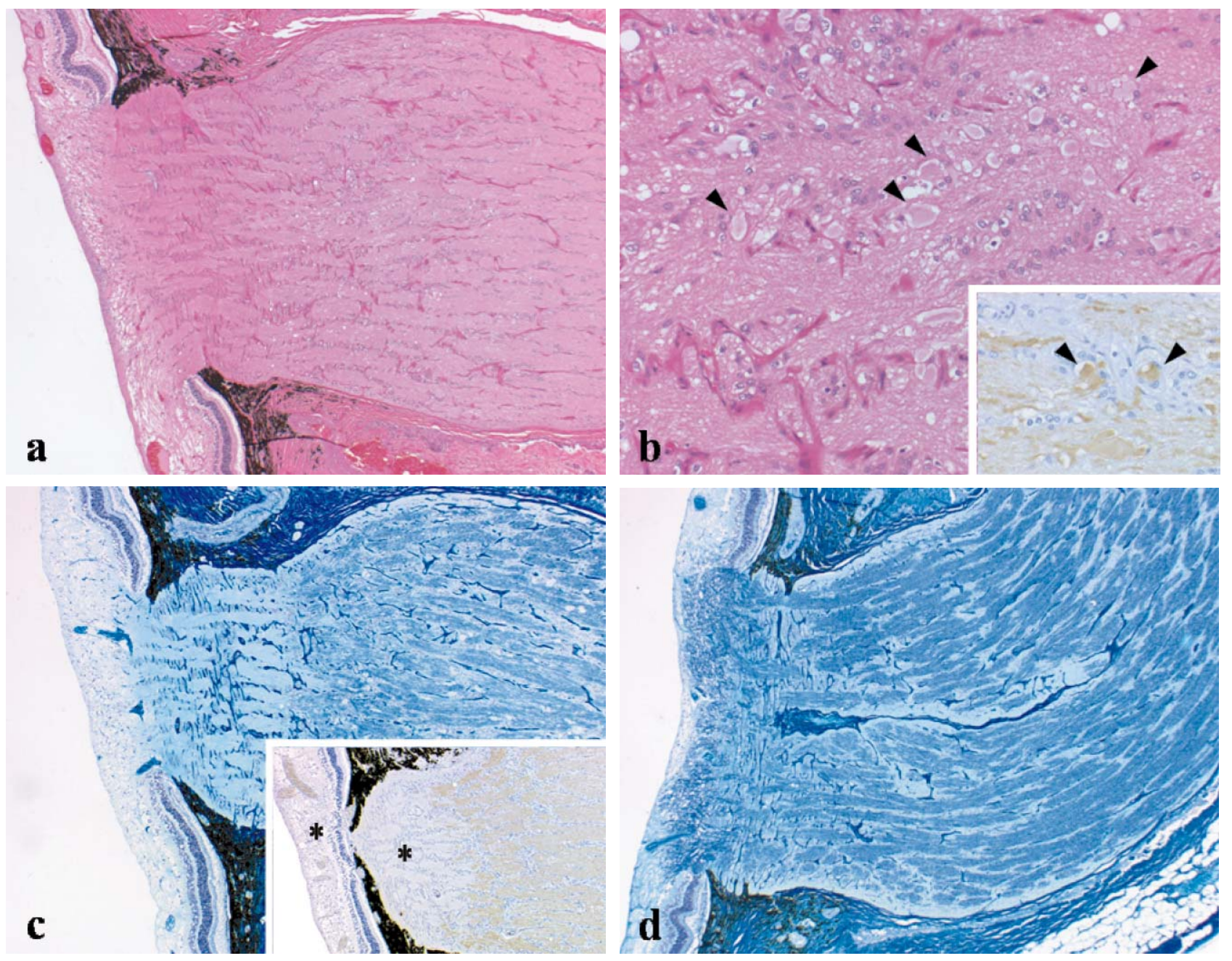

Fig. 2. Optic nerves in a glaucomatous $\operatorname{dog}(a, b, c)$ and a normal dog (d). (a) Glaucomatous cupping of the optic disc is not seen. H\&E stain, $\times 30$. (b) Higher magnification of the optic nerve in the glaucomatous dog shows the presence of spheroid bodies (arrowheads). H\&E stain, $\times 150$. Inset: The spheroid bodies are positive for neurofilament protein. Immunohistochemistry, $\times 150$. (c) The optic nerve head of the glaucomatous dog is poorly stained compared with the normal dog (d). Luxol fast blue stain, $\times 30$. Inset: The optic nerve head of the glaucomatous dog, negative (asterisk) for myelin basic protein. Immunohistochemistry, $\times 25$. (d) The optic nerve head of the normal dog. Luxol fast blue stain, $\times 30$.

2d). Immunohistochemically, the glaucomatous optic nerve head was negative for MBP (Fig. 2c, inset), as was the case for LFB staining. The nerve fiber layer and ganglion cell layer of the retina and optic nerve showed similar reactivity to NF and GFAP in both the glaucomatous and normal dogs.

The dog presented here was diagnosed as having glaucoma due to goniodysgenesis based on histopathological findings such as the absence of a trabecular meshwork. The glaucoma in this dog was thought to be in the early stage based on the animal's clinical course and the degree of degeneration in the optic nerve. Cupping, which is a characteristic finding of glaucoma, was not observed. The histochemical and immunohistochemical findings may be indicative of demyelization in the optic nerve head, although there was no obvious degeneration of the myelin sheaths. In the dog, myelin sheaths cover the axons anterior to the lamina cribrosa, although myelination of the optic nerve head is unusual in other species ${ }^{5}$. This unusual pattern of myelination may be the reason for the resistance of the nerve head to an increase in IOP in the dog. Cupping develops in the dog as the disease progresses, but it is difficult to use this finding as a clinical guide to the progression of glaucoma ${ }^{2}$. Only one half of glaucomatous canine nerve heads were found to show cupping in a histopathologic study ${ }^{6}$.

A mechanical theory that damage is due to an increase in IOP and a vascular theory that damage is due to a reduction in blood flow have been proposed for the pathogenesis of glaucomatous optic neuropathy ${ }^{7}$. In the dog presented here, spheroid bodies (Fig. 2b) indicative of axonal degeneration of the optic nerve were seen in the axons posterior to the lamina cribrosa, but not in the anterior axons. In addition, narrowing of the episcleral veins, fluid retention in the ciliary processes, and retinal edema were observed. These histopathological findings support the 
concept that glaucomatous optic neuropathy may be caused by a reduction in blood flow rather than an increase in $\mathrm{IOP}^{7}$.

The clinical and histopathologic classification of glaucoma in the dog has been confounded by attempts to adapt the descriptive terminology employed in human ophthalmology to the $\operatorname{dog}^{6}$. In the veterinary medical literature, the association of abnormalities in the pectinate ligaments (goniodysgenesis) and angle-closure glaucoma in the dog appears to have similar significance ${ }^{1,2,8}$. In the human, glaucoma due to goniodysgenesis is termed "congenital glaucoma". The term "developmental glaucoma" or "juvenile glaucoma" is used for glaucoma caused by goniodysgenesis developing late in life $e^{4,9,10}$. "Congenital glaucoma" occurs due to underdevelopment of the trabecular meshwork, and in more extensive immature cases, the disease may develop earlier in life ${ }^{10}$. In addition, in the dog, it has been reported that the degree of goniodysgenesis is significantly and positively associated with the likelihood of developing glaucoma ${ }^{11}$. The term "congenital glaucoma" in the dog is commonly used to describe either primary or secondary glaucoma that manifests itself at birth or during the first few months of life $\mathrm{e}^{1,3,8}$. Moreover goniodysgenesis-associated glaucoma in the dog has been classified as a type of primary glaucoma because the clinical signs of glaucoma occur later in life ${ }^{1}$. When glaucoma is caused by goniodysgenesis, as in the dog presented here, we recommend the use of the logical term "goniodysgenetic glaucoma" to clearly indicate the etiology and minimize confusion.

Acknowledgments: The authors would like to thank Mr. S. Shinohara and Mr. S. Ichikawa of Otsuka Pharmaceutical Factory, Inc., for their technical assistance, and Dr. K. Kurisu for his critical review of the manuscript.

\section{References}

1. Gelatt $\mathrm{KN}$ and Brooks DE. The canine glaucomas. In: Veterinary Ophthalmology, 3rd ed., KN Gelatt (ed), Philadelphia, Pennsylvania: Lippincott Williams and Wilkins, 701-754, 1998.

2. Gelatt KN, Brooks DE, and Samuelson DA. Comparative glaucomatology. I: the spontaneous glaucomas. J Glaucoma 1998; 7: 187-201.

3. Peiffer RL Jr, Wilcock BP, Dubielzig RR, Render JA, and Whiteley HE. Fundamentals of veterinary ophthalmic pathology. In: Veterinary Ophthalmology, 3rd ed., KN Gelatt (ed), Philadelphia, Pennsylvania: Lippincott Williams and Wilkins, 355-426, 1998.

4. Tripathi RC, Tripathi BJ, and Haggerty C. Drug-induced glaucomas: mechanism and management. Drug Saf 2003; 26: 749-767.

5. Brooks DE. The canine optic nerve. In: Veterinary Ophthalmology, 3rd ed., KN Gelatt (ed), Philadelphia, Pennsylvania: Lippincott Williams and Wilkins, 981-994, 1998.

6. Smith RIE, Peiffer RL, and Wilcock BP. Some aspects of the pathology of canine glaucoma. Vet Comp Ophthalmol 1993; 3: 16-28.

7. Flammer J, Orgul S, Costa VP, Orzalesi N, Krieglstein GK, Serra LM, Renard JP, and Stefansson E. The impact of ocular blood flow in glaucoma. Prog Retin Eye Res 2002; 21: 359-393.

8. Jones TC, Hunt RD, and King NW. Organs of special sense. In: Veterinary Pathology, 6th ed., Philadelphia, Pennsylvania: Lippincott Williams and Wilkins, 1299-1329, 1997.

9. Jerndal T. Congenital glaucoma due to dominant goniodysgenesis: a new concept of the heredity of glaucoma. Am J Hum Genet 1983; 35: 645-651.

10. Tawara A and Inomata H. Developmental immaturity of the trabecular meshwork in juvenile glaucoma. Am J Ophthalmol 1984; 98: 82-97.

11. Wood JL, Lakhani KH, Mason IK, and Barnett KC. Relationship of the degree of goniodysgenesis and other ocular measurements to glaucoma in Great Danes. Am J Vet Res 2001; 62: 1493-1499. 